\title{
Effects of Pudendal and Cortical Paired Associative Stimulation on Plasticity of Sphincter Responses after Incomplete Spinal Cord Injury: A Feasibility Study
}

\author{
Vásquez $\mathbf{N}^{1 *}$, Knight $\mathrm{S}^{1}$, Susser J ${ }^{1}$, Gall A ${ }^{1}$, Craggs \\ $\mathrm{MD}^{1,2}$ and Ellaway $\mathbf{P H}^{1,3}$ \\ ${ }^{1}$ The London Spinal Cord Injury Centre, Royal National \\ Orthopaedic Hospital, UK \\ ${ }^{2}$ University College London, UK \\ ${ }^{3}$ Imperial College, London, UK \\ *Corresponding author: Natalia Vásquez, London \\ Spinal Cord Injury Centre, Royal National Orthopaedic \\ Hospital, Stanmore, HA7 4LP, UK
}

Received: J une 30, 2017; Accepted: July 24, 2017; Published: July 31, 2017

\begin{abstract}
Objectives: To assess whether paired associative stimulation of the dorsal penile nerve and the motor cortex produces change in anal sphincter muscle responses in incomplete spinal cord injury (iSCl).

Methods: This was a prospective experimental study. Eighteen male iSCl subjects with neuropathic bladder history were recruited. Incontinence was assessed using the International Consultation on Incontinence Modular Questionnaire (ICIQ). Electromyographic activity of the external anal sphincter was recorded. Pudendo-anal reflexes (PAR) were elicited by electrical stimulation of the dorsal penile nerve (DPN) and anal sphincter motor evoked potentials (MEP) by transcranial magnetic stimulation (TMS). Paired associative stimulation (PAS) (DPN and TMS, interval $40 \mathrm{~ms}$ ) was applied for $8 \mathrm{~min}$ at $0.25 \mathrm{~Hz}$ using either real or sham TMS of the motor cortex. Pudendal somatosensory evoked potentials (pSSEPs) were recorded.
\end{abstract}

Results: A PAR could be recorded in all subjects and an MEP in 12 subjects. The PAR was facilitated by prior (30ms) conditioning TMS. Group mean amplitudes of the PAR, the conditioned PAR and MEP, and ICIQ scores showed no change after real or sham PAS. However, 13 subjects individually showed significant changes (increases or decreases) in one or more sphincter responses to real or sham PAS. Individual responses were not correlated with the presence or latency of pSSEPS.

Conclusions: Paired pudendal nerve and cortical stimulation altered the excitability of cortico-spinal and reflex circuitry controlling the anal sphincter in iSCl individuals. Future work should investigate whether such changes in individuals might lead to altered neural circuitry accompanied by functional restoration of continence.

Keywords: Pudendal anal reflex; Sphincter muscle; Motor evoked potential; Paired associative stimulation; Incontinence

\section{Abbreviations}

DPN: Dorsal Penile Nerve; ICIQ: International Consultation On Incontinence Modular Questionnaire; Isci: Incomplete Spinal Cord Injury; MEP: Motor Evoked Potential; PAS: Paired Associative Stimulation; PAR: Pudendo-Anal Reflex; Pssep: Pudendal Somatosensory Evoked Potentials; TMS: Transcranial Magnetic Stimulation

\section{Introduction}

Incomplete spinal cord injury (iSCI) frequently impacts on spino-bulbo-spinal pathways causing major disruption to the control of the pelvic organs and sphincter muscles. Restoration of bladder and bowel control are top priorities for those with iSCI, especially in cases of paraplegia [1]. The neural control involved in bladder and bowel function is complex involving pelvic reflexes, a pontine coordination centre and the sensorimotor cortex. Disruption of spinal pathways can cause neuropathic detrusor over-activity (NDO) leading to detrusor-sphincter dyssynergia. One aspect of NDO and dyssynergia is incontinence resulting from disruption of the guarding reflex and its voluntary control $[2,3]$. This study specifically addresses the possibility of augmenting the guarding reflex in iSCI individuals through plasticity of their residual neural control.

The pudendal anal reflex may be regarded as a surrogate marker for the bladder guarding reflex [4]. In iSCI subjects who retain the reflex, it may be facilitated by conditioning transcranial magnetic stimulation of the motor cortex [5], which is likely to promote continence. This study attempted to establish the feasibility of using repeated, combined stimulation of the pudendal afferent and corticospinal pathways to generate a lasting facilitation through plasticity in the circuits responsible for the guarding reflex.

Repetitive, paired associative stimulation (PAS) [6] involving TMS and peripheral nerve stimulation $[7,8]$ can induce changes in human corticospinal excitability. When a peripheral nerve stimulus was delivered in advance of a cortical TMS pulse with an interval such that the afferent nerve volley arrived at the sensorimotor cortex approximately synchronously with application of the TMS pulse,
Phys Med Rehabil Int - Volume 4 Issue 3 - 2017

ISSN : 2471-0377 | www.austinpublishing group.com

Vásquez et al. () All rights are reserved
Citation: Vásquez N, Knight S, Susser J, Gall A, Craggs MD and Ellaway PH. Effects of Pudendal and Cortical Paired Associative Stimulation on Plasticity of Sphincter Responses after Incomplete Spinal Cord Injury: A Feasibility Study. Phys Med Rehabil Int. 2017; 4(3): 1118. 
Table 1: Demographics and pre-intervention status of subjects.

\begin{tabular}{|c|c|c|c|c|c|c|c|c|}
\hline Subject & AIS grade & Injury Level & Age (y) & Duration of injury (y) & Cause of injury & NDO treatment & ICIQ2 & PAR facilitation \\
\hline 1 & $\mathrm{D}$ & $\mathrm{C} 3$ & 56 & 11 & $\mathrm{~T}$ & Yes & 8 & Yes \\
\hline 2 & $\mathrm{C}$ & $\mathrm{C} 8$ & 62 & 1.5 & $\mathrm{~T}$ & Yes & 7 & Yes \\
\hline 3 & $\mathrm{C}$ & T5 & 43 & 6 & $\mathrm{~T}$ & Yes & 11 & Yes \\
\hline 4 & $\mathrm{D}$ & $\mathrm{C} 5$ & 65 & 7 & non-T & Yes & 7 & Yes \\
\hline 5 & $\mathrm{D}$ & $\mathrm{C} 4$ & 81 & 6 & non- $T$ & No & 4 & Yes \\
\hline 6 & $\mathrm{C}$ & T10 & 65 & 2 & non- $T$ & Yes & 5 & Yes \\
\hline 7 & $\mathrm{D}$ & T10 & 60 & 10 & non- $T$ & Yes & 10 & Yes \\
\hline 8 & $\mathrm{D}$ & $\mathrm{C} 3$ & 52 & 15 & $\mathrm{~T}$ & No & 7 & Yes \\
\hline 9 & $\mathrm{D}$ & T11 & 63 & 21 & $\mathrm{~T}$ & No & 15 & Yes \\
\hline 10 & $C$ & $\mathrm{C} 4$ & 38 & 7 & $\mathrm{~T}$ & Yes & 3 & Yes \\
\hline 11 & $D$ & $\mathrm{~T} 1$ & 67 & 8 & $T$ & No & 1 & Yes \\
\hline 12 & $C$ & L1 & 55 & 30 & $\mathrm{~T}$ & Yes & 12 & Yes \\
\hline 13 & $\mathrm{D}$ & T10 & 46 & 2 & non- $T$ & Yes & 0 & No \\
\hline 14 & $D$ & $\mathrm{~T} 4$ & 50 & 1.5 & non- $T$ & No & 3 & Yes \\
\hline 15 & $\mathrm{D}$ & T4 & 32 & 4 & $\mathrm{~T}$ & Yes & 7 & Yes \\
\hline 16 & $\mathrm{D}$ & $\mathrm{T} 8$ & 57 & 4 & non-T & Yes & 3 & Yes \\
\hline 17 & $\mathrm{D}$ & $\mathrm{C} 4$ & 45 & 1.5 & $\mathrm{~T}$ & Yes & 15 & Yes \\
\hline 18 & C & C3 & 66 & 2 & $\mathrm{~T}$ & Yes & 14 & Yes \\
\hline
\end{tabular}

Subject demographics: Cause of the injury was either traumatic ( $T$ ) or non-traumatic (non-T). Management of neuropathic detrusor over-activity (NDO) employed antimuscarinics or intradetrusor botulinum toxin injection, or some combination of these treatments. The International Consultation on Incontinence Questionnaire (ICIQ2) is the assessment taken immediately prior to the first intervention. PAR facilitation registers whether the PAR could be facilitated by conditioning TMS.

it produced a topographically specific increase in the amplitude of motor-evoked potentials (MEPs) that was long lasting [6].

If a PAS protocol based on paired pudendal nerve and cortical stimulation can be shown to induce plasticity in the cortico-spinal circuits controlling a sphincter muscle [9], a long-term aim would be to investigate whether development of the technique might lead to changes in neural circuitry accompanied by functional restoration of continence.

\section{Methods}

\section{Subjects}

Eighteen males with incomplete, chronic (>1y post injury) and grade $\mathrm{C}$ or $\mathrm{D}$ neurologically-graded supra-sacral spinal cord injuries (American Spinal Injuries Association (ASIA) Impairment Scale (AIS) were recruited following appropriate informed consent (Table 1). All subjects were required to have a history of neuropathic bladder, be able to elicit a weak residual voluntary anal contraction and show a pudendal anal reflex (PAR) in response to electrical stimulation of the dorsal penile nerve (DPN). Some subjects (Table 1) were receiving medical treatment appropriate for their bladder and urethral sphincter dysfunction. Exclusion criteria included lack of tolerance to TMS, contra-indications to TMS (e.g. cranial implants) and concomitant clinical interventions unrelated to the study. Ethics approval for the study was obtained through the UK National Research Ethics Service.

\section{Assessment of incontinence}

The International Consultation on Incontinence Modular Questionnaire (ICIQ) [10] was used to assess urinary incontinence and its impact on quality of life over the previous 4 weeks (21 point scale: 0 = fully continent, 21 maximum incontinence). Subjects completed the ICIQ on four occasions: at recruitment, just before the first intervention (PAS or sham PAS, see below), just before the second intervention and at follow-up.

\section{Electromyography}

The experimental arrangements for recording and stimulating are presented in Figure 1. Electromyography of the external anal sphincter (PAR and MEP) was recorded through an anal-probe electrode (Anuform, Patterson Medical, Sutton in Ashfield, UK). A self-adhesive common reference electrode was attached to the skin of either high. Recordings were amplified (x3000) and filtered (10Hz$2 \mathrm{kHz}$ ) using a CED 1902 isolated preamplifier (Cambridge Electronic Design, Cambridge, UK). Signals were digitally sampled at $4 \mathrm{kHz}$ by a CED1401plus intelligent laboratory interface and analysed using Signal Software (Cambridge Electronic Design, Cambridge, UK) running on a Windows based PC.

\section{Anal sphincter motor evoked potential}

Anal sphincter muscle motor evoked potentials (MEP) were elicited by transcranial magnetic stimulation (TMS) of the motor cortex using a Magstim 200 and a double-cone coil (The Magstim Company, Whitland, UK) [11]. The optimal position of the coil to elicit an MEP was found to be with the cross-over point of the coil within $4 \mathrm{~cm}^{2}$ of the vertex. No MEP could be elicited in 6 of the 18 subjects (Table 2) using the maximal strength of TMS that could be tolerated (usually $70-80 \%$ maximal stimulator output).

\section{Pudendal anal reflex}

Two self-adhesive stimulating electrodes, $20 \mathrm{~mm}$ in diameter 


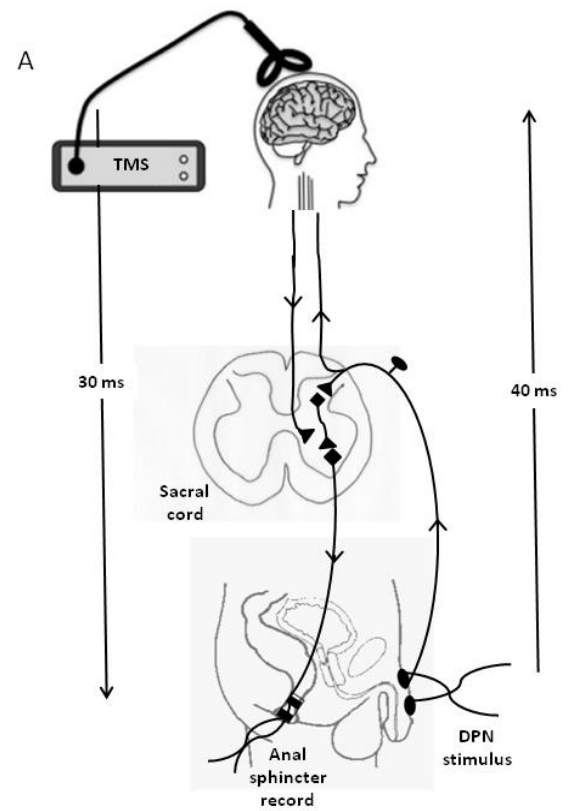

B

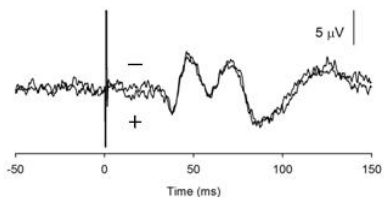

C

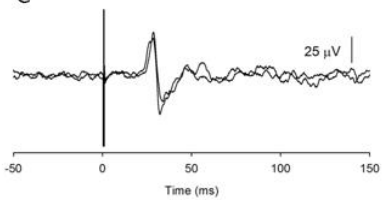

Figure 1: A: Experimental design and schematic of neural pathways. Vertical arrows indicate the approximate time (40 ms) taken for an afferent volley evoked by stimulation of the DPN to reach the somatosensory cortex, and the approximate latency (30ms) of an anal sphincter MEP evoked by TMS of the motor cortex assuming neural transmission unaffected by spinal injury. The average latency of the PAR evoked by stimulation of the DPN (not shown) is $35 \mathrm{~ms}$. B, C: Superimposed averages of pSSEPs (B) and anal sphincter MEP (C) recorded from an iSCl subject (Subject 11 in Table 2). B: Records are averages of 250 unrectified traces to stimulation of the DPN at four times sensory threshold. Latency of the P1 peak is $38 \mathrm{~ms}$. C: records are averages of ten un-rectified traces to TMS at $55 \%$ maximal stimulator output. Latency of the start of the MEP is $25 \mathrm{~ms}$.

(Arbo Neonatal ECG Blue), were attached a few millimetres apart to the skin of the dorsum of the shaft of the penis (Figure 1). Electrical stimuli were delivered by a Digitimer DS7 (Digitimer, Welwyn Garden City, UK) using the proximal electrode as cathode. Sensory perceptual threshold to a single $200 \mu$ s pulse was assessed. Stimulation of the DPN used to elicit a PAR employed a doublet pulse $(200 \mu \mathrm{s}$ pulse duration at $2 \mathrm{~ms}$ intervals), known to improve reproducibility of the reflex [12]. Additionally, the PAR was recorded conditioned (cPAR) by single pulse TMS delivered to the motor cortex $30 \mathrm{~ms}$ before DPN stimulation. This conditioning interval was optimal for facilitating the PAR in control and iSCI subjects [5]. The conditioning TMS was applied just above threshold at the same location used to elicit an MEP or at the maximal tolerable strength of TMS ( 70-80\% maximal stimulator output) in subjects in whom no anal sphincter MEP could be detected.

\section{Somatosensory evoked potential}

Cortical electroencephalography was employed to record pudendal somatosensory evoked potentials (pSSEP) [13]. Electrodes were placed at $\mathrm{Cz}-2 \mathrm{~cm}(\mathrm{Cz})$ (recording electrode) and $\mathrm{Fz}$ (reference electrode) according to the International 10-20 system [14] with a ground electrode placed on one side of the forehead. Electrode impedances were maintained at $<5 \mathrm{k} \Omega$. Recordings used a gain $\times 10000$ and bandwidth $20-2 \mathrm{kHz}$. DPN stimulation was employed using single pulses at 4-5 times perceptual threshold at $3 \mathrm{~Hz}$. Repeated recordings from a total of 250 or 500 pulses were averaged. At least two averages were used to identify features of the pSSEP.

\section{Experimental protocol}

This investigation was a feasibility study but nevertheless modelled as a pilot study based on a single-blinded, sham-controlled, cross-over trial. Subjects received PAS based on DPN stimulation and TMS of the motor cortex. TMS was delivered at the optimal position for representation of the striated muscle component of the external anal sphincter on the motor cortex or over the vertex in subjects lacking an MEP. Subjects also received sham PAS (DPN stimulation and sham TMS component) with an interval of at least two weeks and in a randomised order. Sham stimulation was provided using a circular sham coil placed over the vertex. The stimulation was set to the same intensity for both real and sham stimulation but the sham coil delivered only $5 \%$ of real stimulator output which was insufficient either for MEP generation [15] or excitation of inhibitory circuits in the motor cortex [16]. The sham coil produced an audible click the intensity of which was similar to that generated during real stimulation. The sham element was included in order to be able to account for any effects attributable purely to sensory nerve (DPN) stimulation. Subjects were simply informed that they would receive two types of intervention separated by at least two weeks.

Subjects emptied their bladder just prior to each investigation then lay supine on a bed in a quiet room. The protocol (Figure 1) consisted of a series of threshold determinations (perceptual sensory to DPN stimulation, PAR, MEP) followed by five repeat determinations of the PAR, MEP and cPAR. Subjects were then subjected to a period of PAS consisting of pairs of single pulse DPN stimulation and single pulse TMS with a separation of $40 \mathrm{~ms}$ (DPN before TMS). The repetition rate was $0.25 \mathrm{~Hz}$ and the duration $8 \mathrm{~min}$ (a total of 120 paired stimuli). Alternatively, they were subjected to a similar period of sham PAS.

The DPN stimulus for both the real and sham PAS was adjusted 
Table 2: Summary of individual responses to real and sham paired associative stimulation (PAS).

\begin{tabular}{|c|c|c|c|c|c|c|c|c|c|}
\hline \multirow{3}{*}{ Subject } & \multicolumn{3}{|c|}{ Electrophysiology } & \multicolumn{3}{|c|}{ Response to $1^{\text {stintervention }}$} & \multicolumn{3}{|c|}{ Response to $2^{\text {nd }}$ intervention } \\
\hline & \multirow[t]{2}{*}{ DPN Threshold (mA) } & \multirow[t]{2}{*}{ P1 latency (ms) } & \multirow[t]{2}{*}{ AS MEP latency (ms) } & AS MEP & PAR & CPAR & AS MEP & $P A R$ & $c P A R$ \\
\hline & & & & \multicolumn{3}{|c|}{ Real PAS } & \multicolumn{3}{|c|}{ Sham PAS } \\
\hline 1 & 5 & abs & abs & & & & & & \\
\hline 2 & 7 & 43 & 31 & & & & & & \\
\hline 3 & 7 & abs & abs & & & & & & \\
\hline 4 & 5 & 38 & 25 & & & $\downarrow$ & & & $\downarrow$ \\
\hline 5 & 6 & 43 & 27 & & $\uparrow$ & & $\downarrow$ & & \\
\hline 6 & 5 & 46 & 25 & & & $\uparrow$ & & $\uparrow$ & $\uparrow$ \\
\hline 7 & 16 & abs & abs & & $\uparrow$ & & & $\uparrow$ & \\
\hline 8 & 7 & abs & 29 & & & & & $\uparrow$ & \\
\hline \multirow[t]{2}{*}{9} & 8 & 41 & 23 & $\uparrow$ & & & & & \\
\hline & & & & \multicolumn{3}{|c|}{ Sham PAS } & \multicolumn{3}{|c|}{ Real PAS } \\
\hline 10 & 6 & abs & abs & & & & & $\downarrow$ & $\downarrow$ \\
\hline 11 & 9 & 38 & 25 & & & & & & $\downarrow$ \\
\hline 12 & 12 & abs & 20 & & & & & & $\uparrow$ \\
\hline 13 & 14 & abs & abs & & & & & & \\
\hline 14 & 5 & abs & abs & & & & & $\downarrow$ & \\
\hline 15 & 6 & 40 & 29 & & & $\downarrow$ & & & \\
\hline 16 & 6 & $51^{*}$ & 34 & $\downarrow$ & & & & $\downarrow$ & $\downarrow$ \\
\hline 17 & 8 & $51^{*}$ & 27 & & & & & & \\
\hline 18 & 2 & $48^{*}$ & 30 & & & & & & $\uparrow$ \\
\hline
\end{tabular}

The electrophysiological measures are the threshold ( $T$ ) for perception to electrical stimulation of the dorsal penile nerve (DPN), latency of the P1 wave of the penile somatosensory evoked potential (pSSEP) and latency of the anal sphincter motor evoked potential (AS MEP). A star (*) indicates a latency longer than the normal control subject range. Arrows indicate statistically significant changes in the anal sphincter motor evoked potential (AS MEP) the pudendal anal response (PAR) and the conditioned pudendal anal response (CPAR), ( $\uparrow$ increase, $\downarrow$ decrease) in response to an intervention. Subjects 1-9 received the real PAS first and then the sham PAS. Subjects 10-18 received the interventions in the reverse order.

to a strength that elicited a response approximately one half of the maximum recordable magnitude of the PAR. TMS for the real PAS was adjusted to be just supra-threshold for eliciting an anal sphincter MEP. In subjects in whom no anal sphincter MEP could be detected the strength of TMS that could be tolerated for repetitive stimulation was employed (70-80\% of maximum stimulator output). The PAS interval of $40 \mathrm{~ms}$ was selected with the intention of targeting cortical plasticity. For this to be achieved the afferent impulses excited by DPN stimulation were required to arrive at the sensorimotor cortex approximately synchronous with or a few milliseconds ahead of the application of TMS [8]. Since the peak of the P1 component of the pSSEP recorded in response to DPN stimulation in control subjects has an average latency of $39-40 \mathrm{~ms}$, and is thought to represent cortical activation [17], 40ms was selected as an appropriate PAS interval.

The effect of the PAS (or sham PAS) was immediately assessed by a further five repeat determinations of the PAR, MEP and CPAR. After an additional 20 minutes wait, the assessments were repeated. Following real and sham PAS interventions, at an interval of at least two weeks, a final set of assessments were made. The order in which PAS and sham PAS was administered was randomized and there was at least a two week interval between interventions.

\section{Data analysis}

The time course and magnitude of the PAR, CPAR and MEP were assessed off-line from full-wave rectified averages to ten stimuli repeated on five occasions. Stimulus related responses were identified by averaged signals that exceeded the maximal excursions of an equivalent period of background recording. Two researchers separately inspected recordings and agreed on the presence or otherwise of a response. The magnitude of the response was measured as the area of the response $(\mu \mathrm{V} . \mathrm{ms})$ minus that of a period of background EMG with the same duration occurring prior to stimulation.

In order to assess the effectiveness of conditioning TMS to facilitate the PAR [5], the magnitude of the un-conditioned PAR was taken as the mean value of two recordings made immediately prior to and after the conditioned response. This procedure reduced any impact of adaptation of responses to repeated DPN stimuli.

\section{Statistical analysis}

Paired Student's $t$-test was used to compare differences between PAR and CPAR for individual subjects. Repeated measures one-way ANOVA or the Friedman repeated measures analysis of variance on ranks was used to determine whether an intervention (PAS or sham PAS) caused change in the PAR, cPAR or anal sphincter MEP for either the group or individual subjects. Post-hoc all pairwise multiple comparison procedures (Holm-Sidak) were used to identify any significant result. A $P$ value of $<0.05$ was used to indicate 

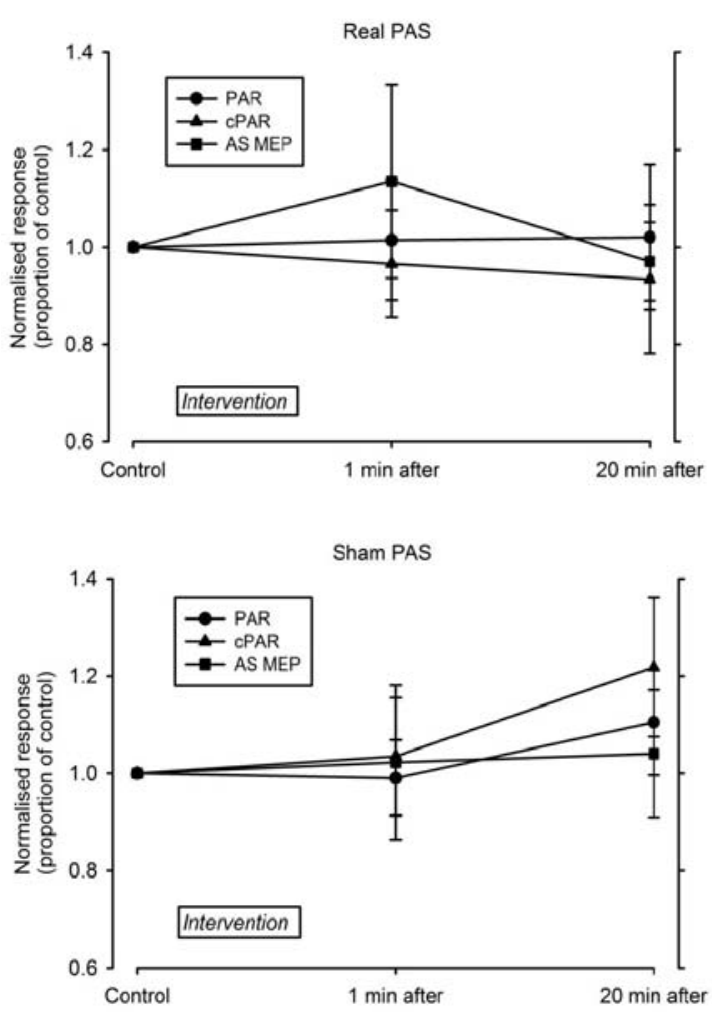

Figure 2: Effects of real and sham PAS interventions on the group of $18 \mathrm{iSCl}$ subjects immediately after the intervention and after a further 20 minutes. Amplitudes of responses are group mean values $( \pm$ SD) normalised to pre-intervention mean values. Top: Real PAS. Bottom: Sham PAS. Subjects are grouped for intervention (real or sham PAS) irrespective of the order in which they received each treatment.

statistical significance for all tests. Statistical tests were carried out using Graphpad Prism (Graphpad Software, Inc. La Jolla, USA) or SigmaPlot 11 software (Systat Software Inc, Chicago).

\section{Results}

\section{Group results}

All subjects showed a PAR in response to DPN stimulation whereas an anal sphincter MEP could be elicited in only 12 of the 18 subjects. The PAR was seen to be facilitated (paired $t$-test, $\mathrm{P}<0.05$ ) when conditioned by a cortical TMS pulse in all but one subject.

For the group of 18 iSCI subjects, neither real or sham PAS intervention caused a statistically significant change in the mean amplitude of the PAR, the CPAR or the anal sphincter MEP either immediately after an intervention or after a further delay of 20 minutes (one-way repeated measures ANOVA, P>0.05) (Figure 2). There was no change in the group mean ICIQ scores assessed at recruitment, immediately before the first intervention (real PAS or sham PAS), before the second intervention and at follow-up (oneway repeated measures ANOVA, $\mathrm{P}>0.05)$.

\section{Individual results}

Of the 18 iSCI subjects, 13 showed significant changes in one or more anal sphincter responses to either real or sham PAS. The results are presented in Table 3 . There were 10 instances of responses being elevated and 11 instances of responses being depressed. Thirteen of the changes were in response to real PAS and 8 in response to sham PAS intervention. There were broadly the same number of changes occurring immediately after PAS $(n=7)$ as after the 20 minute wait $(\mathrm{n}=8)$, with a similar number occurring after PAS and being sustained at 20 minutes $(n=6)$. There were a few instances $(n=3)$ in which the same response was induced both by the real and sham PAS.

Figure 3 illustrates results from the one subject to show an increase in the size of the anal sphincter MEP and results from one of the two subjects who showed an increase in size of the PAR after PAS. In both cases, there was an increase in size of the response in the period immediately after the period of PAS but no significant change from the pre-PAS level after a further interval of 20 minutes.

\section{Afferent pathway}

The outcome of a PAS protocol, namely increasing or decreasing excitability in neural circuits, depends critically on the relative timing of neural volleys $[7,8]$. Since both increases and decreases in excitability were observed in response to PAS it was relevant to assess whether the afferent-cortical interval chosen (40ms) was appropriate in individual subjects. With regard to the afferent pathway, a somatosensory evoked potential could be elicited in 10 of the 18 subjects. Latencies of the peak of the first positive deflection (so-called P1 wave) are given in Table 3. Latencies for three of those subjects (starred) were longer ( $>$ mean $+3 \mathrm{SD}$ ) than expected from a control, neurologically normal population allowing for the height of subjects [18]. Table 3 reveals no particular pattern linking any form of response to PAS (real or sham) either to the presence or absence of a pSSEP, or to the latency of the pSSEP. The intervention (real or sham PAS) significantly changed responses in 8 subjects with a pSSEP, but 

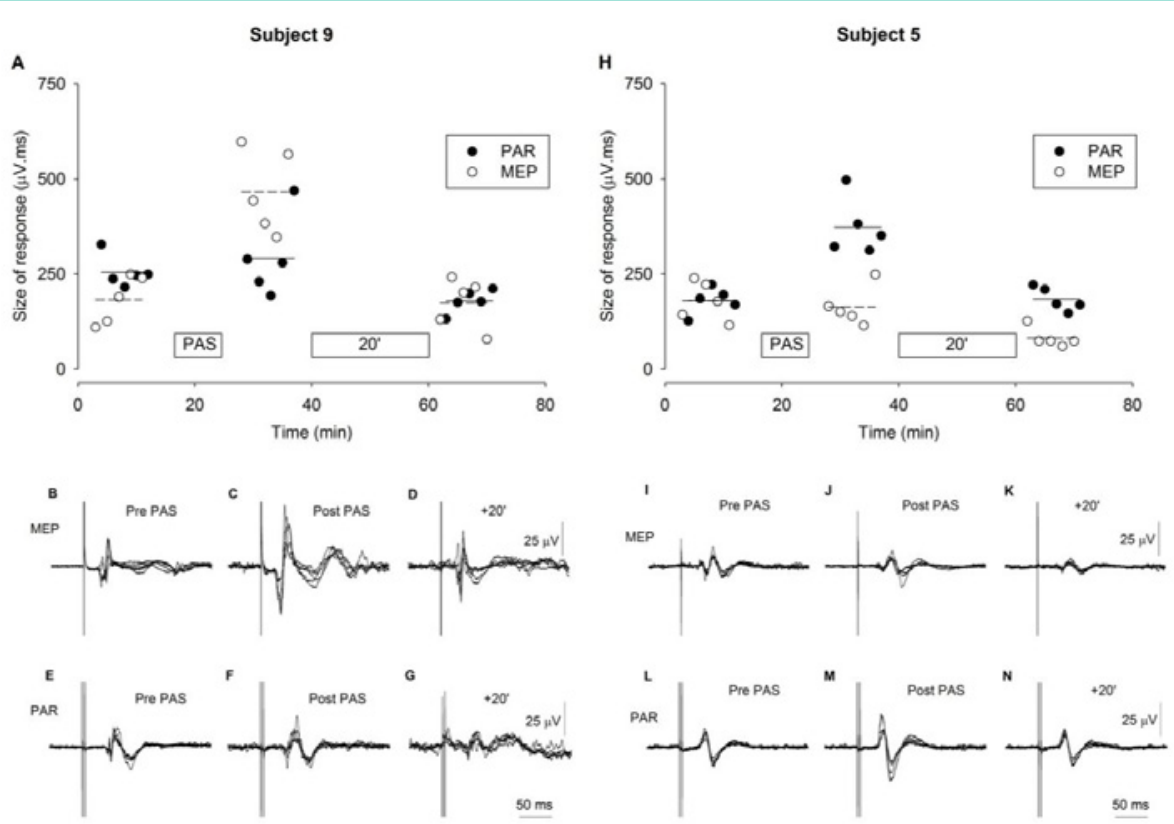

Figure 3: The effect of eight minutes of the PAS intervention on the anal sphincter MEP and PAR responses of two individual iSCl subjects. Neither subject was receiving treatment for NDO. A, H: Size of the anal sphincter MEP (open symbols) and the PAR (closed symbols) before, immediately after and at a further 20 minute after the PAS. Size is a measure of the area of the averaged, rectified response to five repeated stimuli. Mean values of responses are indicated by horizontal lines (MEP, dashed lines; PAR solid lines). There were statistically significant increases immediately after the PAS (repeated measures ANOVA, P<0.05) in the anal sphincter MEP for Subject 9 and in the PAR for Subject 5 but the increases were not sustained at 20 minutes. B-G \& I-N: the traces represent super-impositions of 5 records, each the average of 10 individual stimulations. Stimulation occurs 50 ms after the start of traces. B-D \& I-K: Anal sphincter MEPs. E- G \& L-N: PAR responses. The superimposed traces are from the periods before the intervention (Pre-PAS), immediately after (Post PAS) and after a further 20 minutes (+20').

changes were also observed in 5 subjects who lacked a pSSEP. Two subjects with P1 latencies longer than normal also showed individual responses.

\section{Cortico-spinal pathway}

Latencies of anal sphincter MEPs (Table 3) ranged from 23$33.5 \mathrm{~ms}$ and were all within normal range for control subjects $[19,20]$. There was no statistically significant correlation between pSSEP P1 and MEP latencies (linear regression $\mathrm{r}^{2}=0.26, \mathrm{P}>0.05$ ). However, the observed and expected occurrences (Fisher exact test) of pSSEPs and MEPs were significantly different than expected from random occurrence $(P=0.027)$, i.e. there was a strong tendency for the distribution of the pSSEP and MEP to be linked. All subjects with a pSSEP also had an anal sphincter MEP $(n=10)$ and a further 6 subjects lacked both a pSSEP and MEP. However, responses to PAS (real or sham) were not confined to those subjects with a demonstrable pSSEP and/or MEP.

\section{Discussion}

The results from the group analysis of this study did not reveal any significant overall effect of either the real PAS intervention (paired cortical and pudendal nerve stimulation) or the sham intervention (pudendal nerve stimulation alone) on anal sphincter responses. In contrast, several individuals did show significant changes in cortical or reflex evoked anal sphincter responses to those interventions. However, there was a roughly equal distribution of increased and decreased response amplitudes which may account for the lack of a consistent group response. The explanation for the differences in type of response (or lack of response) to the interventions is possibly related to the indiscriminate nature that the impact of injury has on the spinal cord. Any group of subjects with iSCI can be expected to vary substantially in the impact that injury has on sensorimotor systems and thus potentially on their susceptibility to neural plasticity $[9,21]$.

Functional assessment of urinary continence (ICIQ) also showed no group changes over the period of the investigation. However, since ICIQs assess incontinence over the four week period prior to the assessment [10], the results could not provide an immediate functional outcome to the PAS intervention but merely served to indicate a lack of any functional change in the group over the period of the investigation.

The motivation for considering a PAS protocol in an attempt to induce plasticity in the pathways controlling the anal sphincter was the emerging evidence that the relevant cortical and reflex pathways interact in a facilitatory manner. Voluntary effort facilitates the anal sphincter motor evoked potential [22] and the PAR [23,24] in healthy subjects. Facilitation of the PAR can be induced by conditioning TMS directed at the anal sphincter representation in the motor cortex in neurologically intact subjects and the facilitation is retained in many individuals with iSCI and a neuropathic bladder [5]. These observations indicate a strong facilitatory interaction between cortical and reflex drive of the anal sphincter of relevance to maintaining continence. The PAS protocol used here was devised with the intention of inducing plasticity at the level of the motor cortex with the pudendal stimulation preceding the cortical stimulation by an amount of time adequate in a neurologically normal subject for the afferent volley to have arrived at the time cortical magnetic stimulation was delivered. 
This would have been expected to facilitate cortical excitability $[6,25]$. The results from one subject in the present study (Figure 3, subject 9) fitted this interpretation showing an increase in size of the anal sphincter MEP to the PAS. A pSSEP and an MEP with normal latencies were elicited in that subject. However, several other subjects had similarly normal afferent and motor conduction pathways but failed to show facilitation of the MEP to the PAS.

Two subjects showed facilitation of the PAR rather than the MEP following PAS. One subject (Figure 3 and Table 3, subject 5) had normal pSSEP P1 and MEP latencies, but neither a pSSEP nor an MEP could be elicited in the other subject (Table 3, subject 7). In the case of subject 5 , it is possible that the time window of interaction between individual cortical and pudendal afferent neural actions allowed for induction of temporary plasticity in the spinal circuit mediating the reflex rather than, as had been intended, plasticity at a cortical level [26]. In subject 7, it would be necessary to speculate that residual spinal cord pathways, insensitive to the pSSEP and MEP tests, accounted for the effects of the PAS intervention [9].

A plausible basis for any of the other individual responses is uncertain. Several subjects showed individual changes in sphincter responses to the sham PAS intervention (no TMS component). Induction of plasticity from repetitive stimulation of pudendal afferents alone is not unexpected as there is a substantial literature indicating effectiveness of functional electrical stimulation of pudendal and other sacral afferents in providing restoration of bladder and bowel control in iSCI [30]. Alternatively, it is possible that the electrophysiological tests simply failed to reveal a functional afferent or corticospinal pathway. Residual corticospinal tracts may not be revealed if cortical thresholds for TMS to elicit an MEP in iSCI are high, beyond the acceptable levels of stimulation or the maximum output of the stimulator [9]. False negative pudendal SSEPs have been noted in a study of recovery of bladder function in patients with acute SCI [27] and, indeed, all subjects in the present study could sense the pudendal afferent stimulus albeit with above normal perceptual threshold in some subjects. Recently, neuromodulation of spinal circuitry has resulted in recovery of voluntary movement in individuals with clinically complete motor and sensory paralysis [28] suggesting that residual and latent spinal pathways can affect neural plasticity.

Do the present results provide information that could lead to refinement of the PAS protocol with the intention of augmenting control of sphincter function? Several observations are relevant to answering this question. Transmission time in the afferent pathway activated by pudendal stimulation is an important consideration as the induction of plasticity in corticospinal circuitry is spike timingdependent [29]. Several subjects lacked coherent somatosensory evoked potentials or had significantly delayed P1 waves and showed no change in size of the MEP. In those subjects the cortical afferent input could have been too distributed to interact in a timely manner or would have arrived just after the corticospinal excitation by TMS, possibly even leading to suppression of subsequent cortical excitability [6]. Further development of PAS to evoke plasticity in the neural control of sphincter function in iSCI should at least consider determining afferent conduction time in those individuals demonstrating a clear P1 wave in the dSSEP and adjusting the PAS interval so that an input arrives consistently just before corticospinal stimulation by TMS [8].

A positive aspect of this study is that several individuals did show some ability to respond to interventions designed to induce plasticity in the central nervous system. Given that potential to respond, it will be necessary in carrying this work forward to be selective in the choice of individuals for further interventions based on paired associative stimulation of the motor cortex and pudendal nerves. One approach might be to screen individuals to an initial intervention and then group individuals showing similar responses for repeat trials. No attempt was made to determine whether other factors (duration of injury, NDO medication) influenced outcome of either intervention as group size would have been unacceptably low. Other variables such as extent of the injury (level, severity), urological status and treatment for NDO would also need to be screened in order to group individuals into more closely matched profiles for these future investigations. Additionally, the actual protocol for the PAS intervention should be tailored for individuals taking into account any delays in afferent and efferent pathways caused by the spinal cord injury.

\section{Conclusion}

A paired pudendal afferent and cortical stimulation protocol designed to effect plasticity in anal sphincter responses was effective in individuals but failed to produce a coherent group changes in iSCI subjects with history of neuropathic bladder. Further work should target individuals who show changes to PAS protocols relevant to improved continence through sphincter excitability.

\section{Acknowledgement}

We thank the volunteers who participated in this study.

\section{Funding}

The INSPIRE Foundation (Registered Charity No. 296284 UK) and the RNOH Charity (Registered Charity No. 226955 UK).

\section{References}

1. Anderson KD. Targeting Recovery: Priorities of the Spinal Cord-Injured Population. J Neurotrauma. 2004; 21: 1371-1383.

2. Siroky MB, Krane RJ. Neurologic aspects of detrusor-sphincter dys-synergia with reference to the guarding reflex. J Urol. 1982; 127: 953-957.

3. Park LM, Bloom DA, McGuire EJ. The guarding reflex revisited. Brit J Urol. 1997; 80: 940-945.

4. Podnar S, Vodusek DB. Protocol for clinical neurophysiologic examination of the pelvic floor. Neurourol Urodyn. 2001; 20: 669-682.

5. Vásquez N, Balasubramaniam V, Kuppuswamy A, Knight S, Susser J, Gall A, et al. The interaction of cortico-spinal pathways and the pudendo-anal reflex in patients with incomplete spinal cord injury: A pilot study. Neurourol Urodyn. 2014; 34: 349-355.

6. Stefan K, Kunesch E, Cohen LG, Benecke R, Classen J. Induction of plasticity in the human motor cortex by paired associative stimulation. Brain. 2000; 123: 572-584.

7. Wolters A, Sandbrink F, Schlottmann A, Kunesch E, Stefan K, Cohen LG, et al. A temporally asymmetric Hebbian rule governing plasticity in the human motor cortex. J Neurophysiol. 2003; 89: 2339-2345.

8. Ridding MC, Rothwell JC. Is there a future for therapeutic use of transcrania magnetic stimulation? Nat Rev Neuroscience. 2007; 8: 559-567.

9. Ellaway $\mathrm{PH}$, Vásquez $\mathrm{N}$, Craggs $\mathrm{M}$. Induction of central nervous system 
plasticity by repetitive transcranial magnetic stimulation to promote sensorimotor recovery in incomplete spinal cord injury. Front Integr Neurosci. 2014; 8: 42.

10. Avery K, Donovan J, Peters TJ, Shaw C, Gotoh M, Abrams P, et al. ICIQ: a brief and robust measure for evaluating the symptoms and impact of urinary incontinence. Neurourol Urodyn. 2004; 3: 322-330.

11. Lefaucheur J-P. Excitability of the motor cortical representation of the external anal sphincter. Exp Brain Res. 2005; 160: 268-272.

12. Rodi Z, Vodusek DB. The sacral reflex studies: Single versus double pulse electrical stimulation. Neurourol Urodyn. 1995; 14: 496.

13. Haldeman S, Bradley WE, Bhatia NN, Johnson BK. Pudendal evoked responses. Arch Neurol. 1982; 39: 280-283.

14. Mauguiere F, Allison T, Babiloni C, Buchner H, Eisen AA, Goodin DS, et al. Chapter 2.4 Somatosensory evoked potentials. In, Recommendations for the Practice of Clinical Neurophysiology: Guidelines of the International Federation of Clinical Physiology (EEG Suppl. 52). 1999. Editors: G. Deusch and $\mathrm{A}$. Eisen.

15. Todd G, Flavel SC, Ridding MC. Low-intensity repetitive transcrania magnetic stimulation decreases motor cortical excitability in humans. J App Physiol. 2006: 101; 500-505.

16. Rizzo V, Siebner HR, Modugno N, Presenti A, Munchau A, Gerschlager W et al. Shaping the excitability of human motor cortex with premotor rTMS. J Physiol. 2004; 554: 483-495.

17. Choi YC, Kim YD, Kim WJ, Yang JW, Moon JS. The central conduction time in posterior tibial and pudendal nerve somatosensory evoked potentials. Yonsei Med J. 2001; 42: 9-13.

18. Nikiforidis G, Koutsojannis C, Giannoulis S, Barbalias G. Reduced variance of latencies in pudendal evoked potentials after normalization for body height. Neurourol Urodyn. 1995; 14: 239-251.

19. Pelliccioni G, Scarpino O, Piloni V. Motor evoked potentials recorded from external anal sphincter by cortical and lumbo-sacral magnetic stimulation: normative data. J Neurol Sci. 1997; 149: 69-72.

20. Welter ML, Dechoz S, Leroi AM, Weber J. Evoked mechanical and electrical anal sphincter responses after cortical and lumbar magnetic stimulation. Neurophysiol Clin. 2000; 30: 246-253.
21. Roy FD, Yang JF, Gorassini MA. Afferent regulation of leg motor cortex excitability after incomplete spinal cord injury. J Neurophysiol. 2010; 103: 2222-2233.

22. Loening-Baucke V, Read NW, Yamada T, Barker AT. Evaluation of the moto and sensory components of the pudendal nerve. Electroencephalogr Clin Neurophysiol. 1994; 93: 35-41.

23. Craggs MD, Vaizey CJ. Neurophysiology of the bladder and bowel. In: Fowle CJ, ed. Neurology of Bowel, Bladder and Sex, (Blue Books of Practical Neurology). Oxford: Butterworth-Heinemann; 1999: 19-32.

24. Craggs MD. Pelvic somato-visceral reflexes after spinal cord injury: measures of functional loss and partial preservation. Prog Brain Res. 2006; 152: 205219.

25. Ridding MC, Ziemann U. Determinants of the induction of cortical plasticity by non-invasive brain stimulation in healthy subjects. J Physiol. 2010; 588: 2291-2304.

26. Bunday KL, Perez MA. Motor recovery after spinal cord injury enhanced by strengthening corticospinal synaptic transmission. Curr Biol. 2012; 22: 23552361.

27. Curt A, Rodic B, Schurch B, Dietz V. Recovery of bladder function in patients with acute spinal cord injury: signifcance of ASIA scores and somatosensory evoked potentials. Spinal Cord. 1998; 35: 368-373.

28. Angeli CA, Edgerton VR, Gerasimenko YP, Harkema SJ. Altering spinal cord excitability enables voluntary movements after chronic complete paralysis in humans. Brain. 2014; 137: 1394-1409.

29. Taylor JL, Martin PG. Voluntary motor output is altered by spike-timingdependent changes in the human corticospinal pathway. J Neurosci. 2009; 29: 11708-11716.

30. Grill WM, Craggs MD, Foreman RD, Ludlow CL, Buller JL. Emerging clinical applications of electrical stimulation: opportunities for restoration of function. J Rehabil Res Dev. 2001; 38: 641-653.
Phys Med Rehabil Int - Volume 4 Issue 3 - 2017

ISSN : 2471-0377 | www.austinpublishing group.com

Vásquez et al. () All rights are reserved
Citation: Vásquez N, Knight S, Susser J, Gall A, Craggs MD and Ellaway PH. Effects of Pudendal and Cortical Paired Associative Stimulation on Plasticity of Sphincter Responses after Incomplete Spinal Cord Injury: A Feasibility Study. Phys Med Rehabil Int. 2017; 4(3): 1118. 